\title{
High Temperature Cycles Result in Maternal Transmission and Dengue Infection Differences Between Wolbachia Strains in Aedes aegypti
}

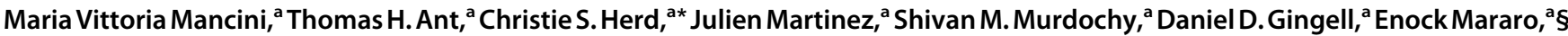 \\ Paul C. D. Johnson, ${ }^{b}$ iD Steven P. Sinkins ${ }^{a}$
}

aMRC-University of Glasgow Centre for Virus Research, Glasgow, UK

bInstitute of Biodiversity, Animal Health and Comparative Medicine, University of Glasgow, Glasgow, UK

M.V.M. and T.H.A. contributed equally to this work. Author order was determined based on higher relative contribution of M.V.M. to experimental planning

ABSTRACT Environmental factors play a crucial role in the population dynamics of arthropod endosymbionts, and therefore in the deployment of Wolbachia symbionts for the control of dengue arboviruses. The potential of Wolbachia to invade, persist, and block virus transmission depends in part on its intracellular density. Several recent studies have highlighted the importance of larval rearing temperature in modulating Wolbachia densities in adults, suggesting that elevated temperatures can severely impact some strains, while having little effect on others. The effect of a replicated tropical heat cycle on Wolbachia density and levels of virus blocking was assessed using Aedes aegypti lines carrying strains wMel and wAlbB, two Wolbachia strains currently used for dengue control. Impacts on intracellular density, maternal transmission fidelity, and dengue inhibition capacity were observed for wMel. In contrast, wAlbB-carrying Ae. aegypti maintained a relatively constant intracellular density at high temperatures and conserved its capacity to inhibit dengue. Following larval heat treatment, wMel showed a degree of density recovery in aging adults, although this was compromised by elevated air temperatures.

IMPORTANCE In the past decades, dengue incidence has dramatically increased all over the world. An emerging dengue control strategy utilizes Aedes aegypti mosquitoes artificially transinfected with the bacterial symbiont Wolbachia, with the ultimate aim of replacing wild mosquito populations. However, the rearing temperature of mosquito larvae is known to impact on some Wolbachia strains. In this study, we compared the effects of a temperature cycle mimicking natural breeding sites in tropical climates on two Wolbachia strains, currently used for open field trials. When choosing the Wolbachia strain to be used in a dengue control program it is important to consider the effects of environmental temperatures on invasiveness and virus inhibition. These results underline the significance of understanding the impact of environmental factors on released mosquitoes, in order to ensure the most efficient strategy for dengue control.

KEYWORDS Aedes, Wolbachia, dengue, mosquito

olbachia are maternally inherited bacterial endosymbionts that naturally infect many arthropod species. The spread through host populations occurs by increasing the relative fitness of carriers in various ways, including reproductive manipulations such as cytoplasmic incompatibility (CI) (1). Cl occurs when a Wolbachia-carrying male mates with a Wolbachia-free female, and results in reduced egg hatching. The major arbovirus mosquito vector Aedes aegypti is not a native Wolbachia host (1); however, artificial transfers have been carried out in the laboratory with a range of
Editor Bruno Lemaitre, EPFL

Copyright $\odot 2021$ Mancini et al. This is an open-access article distributed under the terms of the Creative Commons Attribution 4.0 International license.

Address correspondence to Steven P. Sinkins, steven.sinkins@glasgow.ac.uk.

*Present address: Christie S. Herd, Department of Veterinary Pathobiology, University of Missouri, Columbia, Missouri, USA.

§Present address: Enock Mararo, Ashworth Laboratories, King's Building, University of Edinburgh, Edinburgh, UK.

Received 29 January 2021

Accepted 30 September 2021

Published 9 November 2021 
Wolbachia strains, some of which induce strong $\mathrm{Cl}$ and greatly reduce the competence of Ae. aegypti to transmit arboviruses, including dengue and Zika (2-7). A new dengue control strategy utilizes $\mathrm{Cl}$ to spread Wolbachia through wild mosquito populations and maintain it at high frequency, thereby reducing virus transmission. An increasing number of dengue endemic countries are incorporating releases of Wolbachia-carrying Ae. aegypti as part of ongoing dengue control efforts. Open-field release programs are under way in Indonesia, Vietnam, Australia, Malaysia, Colombia, and Brazil, with significant reductions in dengue incidence reported (8-10). Several Wolbachia strains have been stably introduced into Ae. aegypti, with different strains generating distinct fitness and pathogen blocking profiles. In particular, the Wolbachia strains wAlbB and wMel, native to Aedes albopictus and Drosophila melanogaster, respectively, displayed the most promising characteristics in laboratory studies $(2,5,7,11,12)$ and are both currently being deployed for dengue control.

wMel belongs to the supergroup A Wolbachia clade. It provides protection from RNA viruses in its native host $(13,14)$, and blocks the transmission of dengue (DENV), chikungunya (CHIKV), and Zika (ZIKV) viruses in Ae. aegypti $(2,6,15)$. The wMel infection has been successfully established in Ae. aegypti populations in the cities of Cairns and Townsville in northern Australia, and in Yogyakarta, Indonesia, with data indicating reductions in cases of locally acquired dengue $(9,10,16-18)$. wAlbB belongs to the supergroup B Wolbachia clade, and also efficiently blocks DENV and ZIKV transmission in Ae. aegypti $(3,7,19)$. Open-field releases of wAlbB in Kuala Lumpur, Malaysia, have resulted in high population frequencies and significant reductions in dengue incidence $(8,20)$.

The magnitude of Wolbachia-mediated virus blocking usually shows a positive correlation with intracellular density $(21,22)$. Fitness costs also correlate with density, and high density strains cause both higher fitness costs and strong viral inhibition $(2,7,23-$ $25)$, although there are some exceptions $(7,26)$. wMel and wAlbB reach comparable densities in female Ae. aegypti, and under standard laboratory conditions they show approximately equivalent levels of dengue $(7,11)$ and Zika blocking $(7)$, and both have minimal effects on host fitness $(2,7,27)$.

Invasiveness and stability of a Wolbachia strain depends primarily on $\mathrm{Cl}$ induction capacity, maternal transmission efficiency, and effects on host fitness. The likelihood that a female will mate with a Wolbachia-carrying male and incur the fitness cost of $\mathrm{Cl}$ increases with Wolbachia frequency. The fitness advantage of $\mathrm{Cl}$ is therefore frequency dependent, with invasiveness following bi-stable dynamics determined by an invasion threshold $(28,29)$. Above the threshold the fitness advantages of $\mathrm{Cl}$ overcome other fitness costs and Wolbachia will tend to spread; below the threshold fitness costs dominate and Wolbachia will tend to be lost. The high density wMelPop strain induces strong $\mathrm{Cl}$, but results in fitness costs over a range of life history traits, including reductions in longevity and the survival of eggs following periods of desiccated quiescence. wMelPop carrying Ae. aegypti were released in field sites in Australia and Vietnam and, despite reaching high initial infection frequencies, the strain was eventually lost once releases ceased (30).

Exposure of host insects to thermal stress is known to unbalance and perturb longterm symbiotic interactions and their phenotypes (31, 32), and Wolbachia frequency in insect populations can fluctuate seasonally and between geographical locations (3335). In mosquitoes, several recent studies have demonstrated an impact of larval rearing temperatures on Wolbachia density in the resulting adults, with results suggesting that elevated temperatures can significantly reduce the density of some strains $(7,36$, 37). wMel appears to be particularly sensitive to high temperatures, with density dropping by several orders of magnitude when larvae are exposed to diurnal heat cycling between $27^{\circ} \mathrm{C}$ and $37^{\circ} \mathrm{C}$. In these experiments, a reduced capacity of male carriers to induce $\mathrm{Cl}$ and a lower level of maternal transmission were observed, with eventual loss of the strain when high rearing temperatures were maintained for more than one 


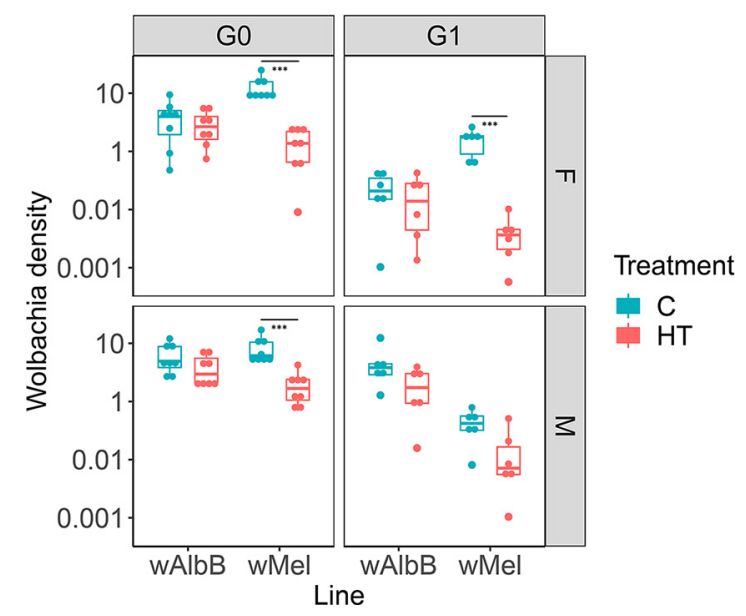

FIG 1 Wolbachia density in whole bodies of control $\left(\mathrm{C}\right.$, constant $\left.27^{\circ} \mathrm{C}\right)$ and heat-treated $(\mathrm{HT}$, temperature $\min =28^{\circ} \mathrm{C}$; temperature $\max =36^{\circ} \mathrm{C}$ ) mosquitoes. The densities of wAlbB and wMel were quantified by qPCR on 5-day-old females (F) and males (M) over two generations of heattreatment. Boxplots represent six biological replicates. Central line indicates the median of densities and whiskers represent upper and lower extremes. T-test and Mann-Whitney test were used for statistical analyses. ${ }^{* *} 0.001$.

generation (36). In contrast, wAlbB was found to be more stable at high temperatures, with little (7) or no (36) reduction in density.

Previous studies have investigated the effects of high larval rearing temperatures on Wolbachia density in whole mosquitoes, and have examined effects on the transmission fidelity $(7,36,37)$. However, reduced densities also suggest the potential for reduced virus blocking. Here, we examine the effects of simulated tropical temperatures on Wolbachia parameters and dengue transmission in transinfected lines of $A$ e. aegypti.

\section{RESULTS}

Effects of field-simulated temperature cycles on Wolbachia density. Detailed temperature recordings from tropical Ae. aegypti larval breeding sites were obtained from a previously published study (38), and a replica-cycle (temperature min: $28^{\circ} \mathrm{C}$; max: $36^{\circ} \mathrm{C}$, Fig. S1) was generated in the laboratory using a programmable dynamic-temperature cabinet (henceforth "heat treatment"). Larvae from wMel- and wAlbB-carrying Ae. aegypti lines were reared under heat or control (constant $27^{\circ} \mathrm{C}$ ) conditions. On eclosion, adult mosquitoes from both treatments were maintained at a constant $27^{\circ} \mathrm{C}$. Five-day-old adults were sacrificed, and Wolbachia densities assessed (Fig. 1). Subsets of females were blood-fed, and the resulting progeny exposed to a second round of larval heat treatment, with Wolbachia densities in $\mathrm{G} 1$ adults assessed 5 days after emergence. Consistent with previous studies $(7,36,37)$, the wMel strain was particularly susceptible to density reductions resulting from heat treatment, with a significant drop in density in females from $12.6 \pm 5.9 \mathrm{Ct}$ wsp/Ct HTH (mean \pm SD) to $1.4 \pm 0.9 \mathrm{Ct}$ wsp/Ct HTH $(P<0.001$, Mann-Whitney test) after one generation of heat treatment. These results confirm that the density drop did not result from a sudden heat shock resulting from the two-temperature diurnal regime used in previous studies. A similar trend was observed in female adults resulting from a second larval heat treatment, decreasing from $1.5 \pm 0.7 \mathrm{Ct}$ wsp/Ct HTH to $0.04 \pm 0.03 \mathrm{Ct}$ wsp/Ct HTH ( $P<0.001$, unpaired $t$ test). WMel density decreased also in male adults, significantly dropping after one round of larval heat treatment $(P<0.001$, Mann-Whitney test); in contrast, the second larval heat treatment on the male progeny had a milder effect, causing a slight decrease (not significant, $P=0.06$ ) in wMel density. The wAlbB strain maintained a constant density over both generations of heat treatment in females and males $(p>0.5$ for both generations and sexes, Unpaired $t$ test and Mann-Whitney test). 

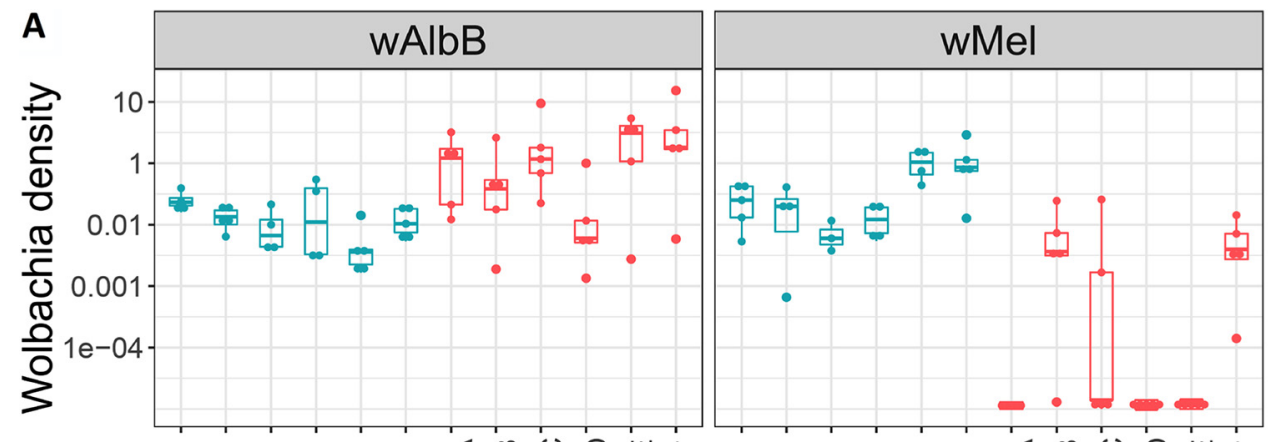

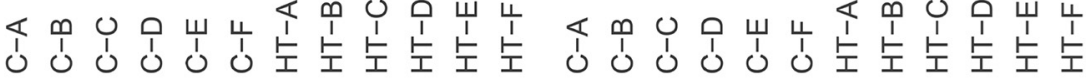

B

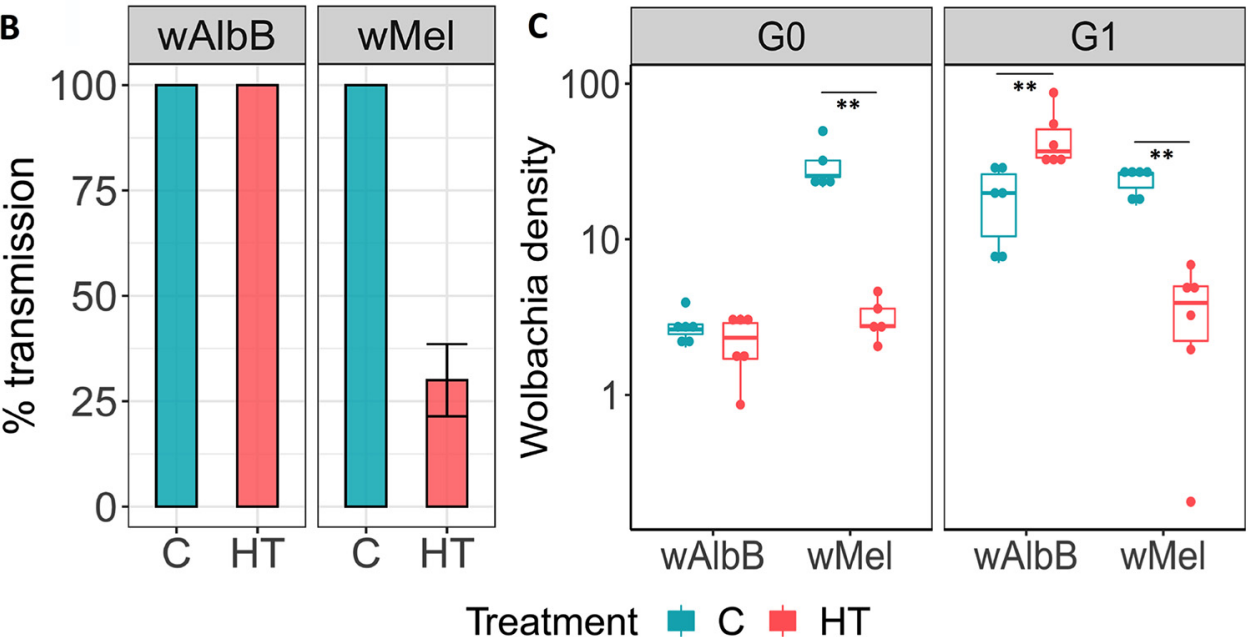

FIG 2 Whole-body densities, maternal transmission rate, and ovary-specific densities of $w A$ lbB and $w$ Mel in control $\left(\mathrm{C}\right.$, constant $\left.27^{\circ} \mathrm{C}\right)$ and heat-treated $\left(\mathrm{HT}\right.$, temperature min $=28^{\circ} \mathrm{C}$; temperature $\left.\max 36^{\circ} \mathrm{C}\right)$ mosquitoes. (A) Progeny from single females reared as larvae under $C$ or $H T$ conditions were hatched in families $(A, B, C, D$, $\mathrm{E}, \mathrm{F})$ and reared at $27^{\circ} \mathrm{C}$. Six L4 larvae were randomly sampled from each individualized female and assessed for Wolbachia density by qPCR (A) and infection-status by strain-specific PCR $(N=60$ for each treatment/ strain). (B). Wolbachia density data were computed using linear mixed models with fixed (Wolbachia line, treatment) and random effect (families), followed by multiple comparisons and $P$ values adjustment. (C) Densities of wAlbB and wMel were measured in six pools of three sets of dissected ovaries. The center of a box indicates the median of densities and whiskers represent upper and lower extremes. A Mann-Whitney test was used. ${ }^{* *} 0.01 ;{ }^{*} 0.05$.

Effects of field-simulated temperature cycles on Wolbachia maternal transmission. Wolbachia-carrying females reared under either heat treatment or control conditions were back-crossed to wild-type males. Females were individualized for oviposition and the resulting $\mathrm{G} 1$ eggs hatched as single families. G1 larvae were reared at a constant $27^{\circ} \mathrm{C}$ until the L4 stage, whereupon a random selection from each family was assessed for Wolbachia status and density. Comparisons between models showed a significant effect of the Wolbachia strain $\left(P<0.001, \chi_{\mathrm{df}=1}^{2}=18.2\right)$ and of the interaction between the strain of Wolbachia and the high-temperature larval treatment $\left(P<0.001 \chi^{2}{ }_{\mathrm{df}=3}=\right.$ 67.6). wMel densities in the Wolbachia positive G1 progeny were significantly lower following heat treatment than densities in the $\mathrm{G} 1$ progeny following control treatment $(p<0.001)$ (Fig. 2A); endpoint PCR showed that the maternal transmission of wMel was significantly reduced following larval heat treatment, with the complete loss of wMel in three of the six heat-treated families, compared with $100 \%$ transmission in the control group ( $P<0.001$, Fisher's exact test) (Fig. 2B).

In contrast, $w A$ IbB females resulting from larvae reared under either heat treatment or control conditions transmitted Wolbachia to $100 \%$ of offspring. Interestingly, the G1 progeny from heat treated $w A$ AlbB mothers displayed higher Wolbachia densities compared to progeny resulting from mothers reared under control conditions $(P<0.001)$. 

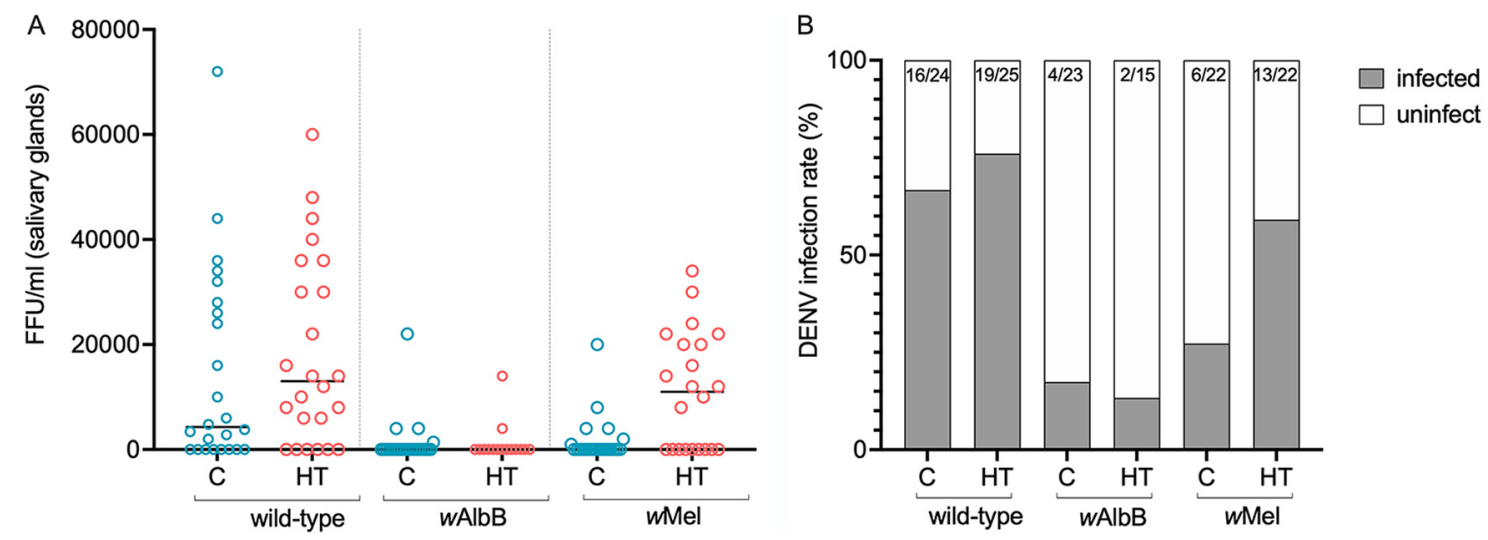

FIG 3 Effect of larval heat-treatment on dengue inhibition. Wild-type, wAlbB-, and wMel-carrying females were fed on DENV2infected blood-meal. Engorged females were selected and incubated for 12 days. Salivary glands from control and heat-treated females were assessed for virus dissemination by FFA. Dots represent the number of foci/ml and each dot corresponds to a single mosquito (A). Dissemination rates from the same experiments are represented in panel B. Proportions on each bar indicate the infection rates ( $N$ positive/ $N$ total). Statistical analysis was performed using a generalized linear model.

A strong significant difference in Wolbachia load was also observed between the progeny of $w$ AlbB and wMel heat-treated females $(P<0.001)$ (Fig. 2A).

To associate perturbations in maternal transmission with Wolbachia densities in ovaries, females reared under either heat treatment or control conditions were dissected, and ovary densities assessed by qPCR. Results indicate that heat treatment caused significant reductions in the ovary density of wMel, while the density of wAlbB was not negatively affected, and even increased ( $P=0.002$, Mann-Whitney test), compared with controls following two generations of treatment (Fig. 2C). Effects on Wolbachia density in mosquito ovaries can also be visualized by whole-mount fluorescent in situ hybridization (FISH) (Fig. S2).

Effects of field-simulated temperature cycles on virus transmission. To test whether temperature-induced changes in the Wolbachia lines (assumed to be associated with Wolbachia density changes) could impact dengue inhibition, larvae from the $w A l b B, w M e l$ and wild-type lines were reared under either heat treatment or control conditions, and the resulting 5-day old adult females were orally challenged with a bloodmeal containing DENV-2. Twelve days postfeeding, levels of infectious virus in mosquito tissues were quantified to assess infection and transmission potential within the vector (Fig. 3, Fig. S3).

We tested the effects of strain, heat treatment, and their interaction on both the probability of viral infection and the intensity of infection (viral load) given that infection had occurred. The ability of wMel to affect viral infection intensity was dependent on whether heat treatment or control larval rearing was used $\left(P=0.048, \chi_{\mathrm{df}=1}^{2}=3.92\right)$. wMel mosquitoes displayed significantly lower viral infection titer compared with wildtype when reared at standard control conditions $\left(P=0.005, \chi_{\mathrm{df}=1}^{2}=7.98\right)$, but there was a difference between the strains when reared under heat treatment $(P=0.365$, $\chi_{\mathrm{df=1}}^{2}=0.82$ ) (Fig. 3A). In addition, wMel mosquitoes showed a higher probability of infection inhibition than wild type irrespective of larval rearing temperature $(P=0.006$, $\chi_{\mathrm{df}=1}^{2}=7.59$ ) —only six mosquitoes out of 22 were found positive for DENV viral particles (27\%) (Fig. 3A and B). There was also an increased probability of infection in the heat treatment cohort regardless of strain $\left(P=0.047, \chi_{\mathrm{df}=1}^{2}=3.95\right)$ : $59 \%(13 / 22)$ of heattreated mosquitoes were found to be infected (Fig. 3B).

wAlbB maintained strong viral inhibition following high-temperature rearing, showing a highly significant effect of the strain on viral infection intensity and prevalence, compared with the wild-type group (Fig. $3 \mathrm{~A}$ and $\mathrm{B}$ ). wAlbB had a negative effect on viral load in salivary glands $\left(P=0.020, \chi_{\mathrm{df}=1}^{2}=5.42\right)$ regardless of larval rearing regime, 
and significantly decreased the probability of infection $\left(P<0.001, \chi_{\mathrm{df}=1}^{2}=27.70\right)$, with the odds ratio for infection relative to the wild-type control of 0.077 .

From an independent viral challenge, a similar pattern of infection was observed in the salivary glands of heat-treated mosquitoes (Fig. S3). wMel females displayed a significant increase in viral titer (Fig. S3 A) and in infection rate (Fig. S3 B), compared with heat-treated wAlbB $(P<0.001$ Mann-Whitney test; $P<0.001$, Fisher's exact test, respectively). While viral titers in salivary gland tissue were significantly lower in hightemperature-reared wAlbB females compared to wild-type females $(P=0.006$ MannWhitney test), no significant difference was observed between heat-treated $w$ Mel and wild-type females ( $P=0.275$, Mann-Whitney test).

Adult exposure to elevated temperatures and Wolbachia recovery. A previous study reported substantial recovery of wMel in adult Ae. aegypti from initially low densities following larval rearing at high temperatures (37). To further investigate density recovery in adults, and to examine the impact of elevated air temperatures on recovery rates, wMel and wAlbB larvae were reared under control or high temperature conditions, with emerging adults exposed to replica heat cycles generated from recordings of ambient temperatures in shaded (henceforth "adult temperature regime 1"-temperature min: $28^{\circ} \mathrm{C}$; max: $33.5^{\circ} \mathrm{C}$ ) or semi-shaded (henceforth "adult temperature regime $2^{\prime \prime}$-temperature min: $27^{\circ} \mathrm{C}$; max: $36.5^{\circ} \mathrm{C}$ ) sites in urban Kuala Lumpur (Fig. S1). Adult females were dissected, and Wolbachia densities in midgut and salivary gland tissues were assessed (Fig. 4).

There was a significant reduction in the density of wMel in adults emerging from heat treatment $(0.02 \pm 0.01 \mathrm{Ct}$ wsp/Ct HTH, mean $\pm \mathrm{SD})$ compared with control treatments $(2.8 \pm 2.6 \mathrm{Ct}$ wsp/Ct HTH $)(p<0.001$, Mann-Whitney test). However, there was a marked recovery in density in heat treated larvae subsequently reared under control conditions as adults (reaching $0.4 \pm 0.4 \mathrm{Ct}$ wsp/Ct HTH after 14 days), although this recovery was incomplete, with adults from control larvae maintaining a significantly higher density, $5.9 \pm 3.7 \mathrm{Ct}$ wsp/Ct HTH after 14 days ( $p=0.003$, Mann-Whitney test). Air temperature had a significant impact on the recovery of wMel, with 14-day-old females from the adult regimes 1 and 2 displaying significantly lower densities than adults reared at control temperatures $(p<0.001$ for both adult temperature regimes, Mann-Whitney test).

A similar trend was observed in dissected salivary gland and midgut tissues of emerging adults, with significant reductions in wMel density in both tissues following larval heat treatment $(p<0.005$ for both midguts and salivary glands, Mann-Whitney test). There was a recovery in density in midguts at day 14 in the heat treatment cohort reared at the control adult temperature, with no significant difference compared to mosquitoes reared exclusively under control conditions ( $P=0.48$, Mann-Whitney test). Densities in the salivary glands of females reared under heat treatment showed minimal recovery at both control and adult temperature regime 1, with significantly lower densities compared with mosquitoes reared exclusively under control conditions ( $p<$ 0.005 for both control and adult temperature regime 1, Mann-Whitney test). Adults maintained under temperature regime 2 showed significant reductions in density in both salivary gland and midgut tissues compared with adults reared at control temperatures ( $p<0.005$ for both salivary glands and midguts, Mann-Whitney test).

$w A l b B$ showed a reduction in density in adults emerging from heat treatment $(0.4 \pm 0.2 \mathrm{Ct} \mathrm{wsp} / \mathrm{Ct} \mathrm{HTH})$ compared with control treatments $(1.6 \pm 0.4 \mathrm{Ct}$ wsp/Ct HTH) ( $p<0.001$, Mann-Whitney test). However, the density recovered fully after 7 days of adult rearing under control conditions, with no significant reduction in density compared to mosquitoes reared only at control temperatures $(P=0.129$, Mann-Whitney test). Interestingly, wAlbB-carriers reared at the control temperature as larvae and the adult temperature cycle 1 showed significantly increased Wolbachia densities compared with adults reared only under control conditions ( $P=0.002$, Mann-Whitney test). Adult treatment 2 resulted in significant reductions in densities compared with 

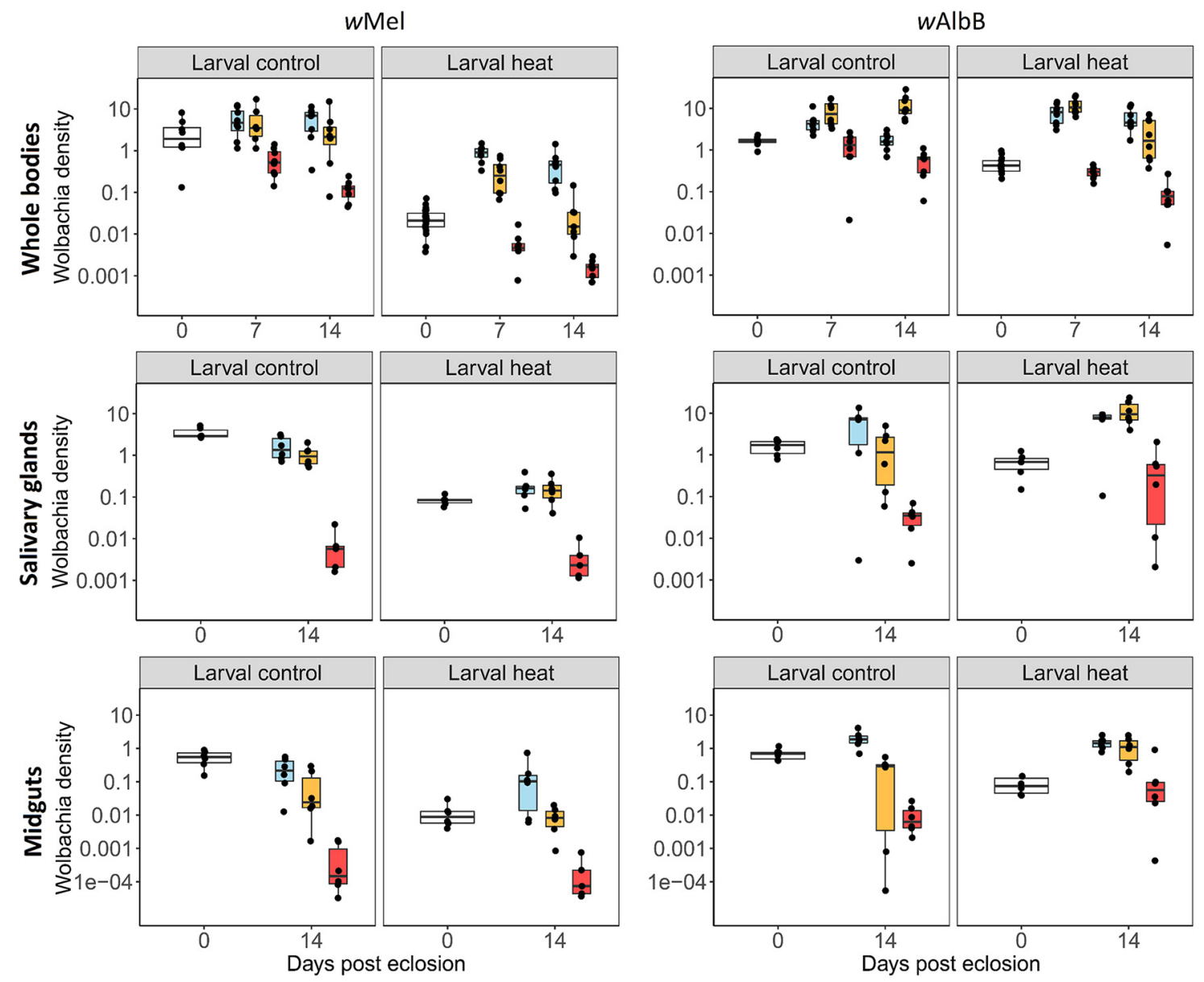

혀 Control 혀 Heat treatment 1 후 Heat treatment 2

FIG 4 Effects of high temperature larval and adult ambient air temperatures on wMel and wAlbB densities in whole-bodies, salivarygland, and midgut tissues. Larvae were reared under control (larval control, constant $27^{\circ} \mathrm{C}$ ) and high temperature (larval heat, temperature $\min =27^{\circ} \mathrm{C}$; temperature $\max 37^{\circ} \mathrm{C}$ ) conditions. A subset of females was sampled immediately on eclosion (day 0 ), and the densities assessed. The remaining females were divided into three adult treatment temperatures: control (constant $\left.27^{\circ} \mathrm{C}\right)$, adult regime 1 (temperature $\min =28^{\circ} \mathrm{C}$; temperature $\max =33.5^{\circ} \mathrm{C}$ ), and adult regime 2 (temperature min $=27^{\circ} \mathrm{C}$; temperature max $=36.5^{\circ} \mathrm{C}$ ). Adults were sampled and densities assessed in whole bodies (days 7 and 14 posteclosion) and dissected salivary gland and midgut tissues (day 14 posteclosion). Data points represent single whole adult females, or pools of three salivary glands or midguts.

adults reared at the control temperature, regardless of larval treatment ( $p<0.001$ for both control and heat-treated, Mann-Whitney test).

Following larval heat treatment, the density of $w A$ lbB in both midgut and salivary gland tissues of eclosing adults was slightly but significantly reduced $(P=0.02$ for salivary glands; $P=0.002$ for midguts). However, densities in both tissues recovered fully at day 14 when adults were reared under either control or adult temperature regime 1 temperature conditions, displaying no significant reductions compared to tissue densities in adults reared under control conditions only ( $p>0.18$ for all comparisons). Similar to $w$ Mel, rearing $w A$ AlbB adults under the temperature regime 2 resulted in significant reductions in densities compared to adults reared under control conditions, regardless of larval treatment.

\section{DISCUSSION}

Ae. aegypti larvae developing in the tropics encounter a far broader and variable range of temperatures than those typically used in mosquito insectaries (usually stringently maintained in the range of $27^{\circ} \mathrm{C}-28^{\circ} \mathrm{C}$ ). Several recent studies have highlighted 
the substantial influence that larval water temperature has in determining the density of some Wolbachia strains in Ae. aegypti, particularly wMel $(36,37,39)$. This is noteworthy as Wolbachia strain characterization is routinely performed under standard insectary temperatures and suggests that phenotypes predicted by laboratory tests may vary in the field. Tropical breeding sites can experience heating above $30^{\circ} \mathrm{C}$ for extended periods of the day, and in some cases reach daily maxima in excess of $36^{\circ} \mathrm{C}$ $(38,40)$. The high temperature regime used in this study was generated from data collected from water drums in Trinidad acting as Ae. aegypti larval habitats (38).

Consistent with previous studies, wMel was found to be negatively affected by exposure to the high temperature cycle, showing a significant decrease in whole body density. A substantial drop in adult ovary density was also observed, leading to a reduction in maternal transmission of approximately $75 \%$. Imperfect maternal transmission can impact the population stability of a Wolbachia infection by increasing the invasion threshold, potentially compromising the ability of wMel to spread and persist in wild populations. Previous evidence documented a disruption in wMel maternal transmission and $\mathrm{Cl}$ induction following exposure to high temperatures $(7,36)$. Additionally, intense artificial laboratory selection for a heat resistant wMel variant in Ae. aegypti failed to produce a strain with improved thermal tolerance, an observation that was supported by experiments showing that field collected wMel-carriers from a hot climate did not differ substantially in their response to heat stress compared with a laboratory colony-suggesting that adaption of the strain to high temperatures may be intrinsically difficult (39). In contrast, the wAlbB strain showed relative heat stability when larvae were reared under the high temperature regime. High densities were maintained in the ovaries, resulting in complete maternal transmission, suggesting that the wAlbB strain would be more stable among populations in hot tropical climates.

For the first time, the consequences of tropical heat stress on the ability of Wolbachia to inhibit dengue virus dissemination was tested in wMel and wAlbB-carrying Ae. aegypti. Rates of mosquito infection following challenge with DENV2 were quantified in order to predict the infective state of mosquitoes reared under either heat treatment or control conditions. Following exposure to the thermal stress of heat treatment conditions, wAlbB retained its ability to efficiently block DENV2 dissemination, while wMel showed a significant increase in viral dissemination. Wolbachia-mediated viral inhibition is thought to be primarily cell autonomous $(5,41)$; consequently, densities in midgut and salivary gland tissues are key to blocking virus dissemination and transmission. The reduction in dengue inhibition in heat-treated wMel is concomitant with large reductions in Wolbachia density in both midgut and salivary gland tissues, although the density in midguts appeared to recover in adults after 14 days. WAlbB also showed reductions in density in midgut and salivary gland tissues, although the reduction was not as dramatic as wMel, and recovered fully in 14-day-old adults.

A decrease in the efficiency of dengue blocking by wMel could have significant impacts on the utility of the strain as a vector control intervention in hot tropical climates. This is particularly relevant given the role of high temperatures as a covariate of dengue transmission (42). Moreover, there is the potential that a weakening of the wMel transmission blocking phenotype following exposure to high temperatures could increase the risk of selection of virus escape mutations that confer a lower general susceptibility to Wolbachiamediated inhibition-and could therefore undermine Wolbachia interventions. It should be noted that Wolbachia at high density induce a broad range of perturbations in $A e$. aegypti cells (43), including in a number of pathways that are important in the flavivirus life cycle-such as lipid transport and metabolism, autophagy, vesicular trafficking, and endoplasmic reticulum stress; this is inherently likely to reduce the risk of selection of virus escape mutations. However, at lower density the levels of perturbation are reduced (43).

A previous study has shown that initially low densities of wMel following larval heat treatment can recover substantially in adults reared under normal insectary conditions (37). Results presented here are consistent with this finding, with wMel showing considerable (although incomplete) density recovery when adults are reared at a constant 
$27^{\circ} \mathrm{C}$. However, while adult mosquitoes are able to fly and seek cooler resting areas, ambient air temperatures are often very high in the tropics. Recordings in shaded and semishaded sites from urban Kuala Lumpur indicate that air temperatures can reach in excess of $34^{\circ} \mathrm{C}$ for several hours of the day. In larvae carrying wMel reared using the high temperature cycle, and subsequently reared as adults using a replica shaded airtemperature cycle (defined as adult temperature regime 1), only a limited recovery in Wolbachia density occurred. In contrast, the density of wAlbB in whole mosquitoes reared as adults using the temperature cycle 1 were significantly higher than controls - suggesting that the temperature optimal for wAlbB replication may actually be higher than the $27^{\circ} \mathrm{C}$ used in standard rearing. Both wMel and wAlbB densities in adults were substantially reduced by exposure to the temperature cycle 2 , suggesting that $w A l b B$ is not completely resistant to the effects of high temperatures, although this cycle represents a temperature regime that adult mosquitoes will be unlikely to encounter for extended periods. The wAlbB strain was capable of reaching and maintaining high frequencies and significantly reducing dengue transmission in the hot tropical climate of urban Kuala Lumpur, Malaysia (8).

Although the wMel strain used in this study shows reduced densities in the laboratory using simulated field conditions, releases in Australia, Brazil, and Indonesia demonstrate that wMel can stably invade wild Ae. aegypti populations (9, 10, 16, 44, 45) and maintain its ability to block dengue $(17,46)$. In direct comparisons, wMel line produced slightly lower fitness costs than wAlbB (7), suggesting that it may be the more invasive of the two strains in cooler climates. Exposure of wMel-carrying Ae. aegypti adults to a diurnal temperature cycle with a mean of $28^{\circ} \mathrm{C}$ and a fluctuating range of $8^{\circ} \mathrm{C}\left( \pm 4^{\circ} \mathrm{C}\right)$ caused a decrease in bacterial density when compared to constant $25^{\circ} \mathrm{C}$, but did not reduce the ability of Wolbachia to inhibit dengue transmission (47). Moreover, in some hotter equatorial locations Ae. aegypti can exploit underground larval habitats, such as wells and drains, which will be away from direct sunlight and cooler than ground level. Laboratory experiments have also proved that the effects of thermal stress on Wolbachia density are stage-specific $(35,37)$; in particular, exposure of early larval stage generates a significant and irreversible decrease in density, while the drop observed during exposure to later stages is rescued during adulthood. This suggests that the variations in temperature typical of the field will result in a more complex gradient of phenotypes, less clear-cut than those produced in the laboratory. The complex interactions between environmental temperatures and Wolbachia phenotypes has been recently investigated in natural Wolbachia-Drosophila associations, where the developmental temperature of the host modulated Wolbachia-induced antiviral effects, ranging from complete to no protection, affecting Wolbachia density (higher at $25^{\circ} \mathrm{C}$ compared with $18^{\circ} \mathrm{C}$ ); in contrast, postinfection exposure to different temperatures impairs viral protection without affecting symbiont density (48).

Our data confirm that high tropical temperatures have a significant impact on the phenotypic stability of Wolbachia in Ae. aegypti, and the magnitude of this impact varies substantially between Wolbachia strains. Of the strains currently used in open field releases, wMel appears to be particularly susceptible and wAlbB relatively stable under thermal stress, with wMel displaying a marked reduction in capacity for maternal transmission and dengue blocking - which is not observed with wAlbB. The selection for the optimal strain for Wolbachia-deployed vector control strategies must therefore consider phenotypic stability in relation to the geography and climate of selected intervention areas. The water temperature of natural breeding sites not only represents a crucial abiotic factor known to directly affect vector biology (49), but it also plays a role in ensuring the most effective Wolbachia-based strategy for reducing dengue transmission.

\section{MATERIALS AND METHODS}

Mosquito rearing. $w \mathrm{Mel}, w \mathrm{AlbB}$, and wild-type Ae. aegypti mosquitoes were derived from previously generated lines (7), sharing the same genetic background. Colonies were maintained at $27^{\circ} \mathrm{C}$ and $70 \%$ relative humidity with a 12 -h light/dark cycle. Larvae were fed with tropical fish pellets (Tetramin, Tetra, 
Melle, Germany) and adults maintained with $5 \%$ sucrose solution ad libitum. Blood meals were provided using an artificial blood-feeding system (Hemotek, UK) using human blood (Scottish National Blood Transfusion Service, UK). Eggs were collected on a wet filter-paper (Grade 1 filter paper, Whatman plc, GE health care, UK). Eggs were desiccated for 5 days and later hatched in deionized water containing $1 \mathrm{~g} / \mathrm{L}$ bovine liver powder (MP Biomedicals, Santa Ana, CA, USA).

Temperature cycles. For each replicate performed over multiple generations, eggs from wMel, wAlbB, and wild-type (WT) Ae. aegypti lines were hatched and separated into experimental groups: larval density (200 larvae per $500 \mathrm{ml}$ of water) and food were consistent between the conditions.

Heat-challenged larvae were maintained in Panasonic MLR-352-H Plant Growth Chamber incubator (Panasonic, Osaka, Japan). The applied temperature regime was based on data from Ae. aegypti larval breeding containers in Trinidad (38) and replicated in the cabinets. Water temperatures were continuously monitored using a data logger (Hobo Water Temperature Pro V2, Bourne, MA, USA) placed in a plastic tray filled with $500 \mathrm{ml}$ of water. Temperature data were registered and monitored. Mosquitoes under control conditions were stably maintained at $27^{\circ} \mathrm{C}$, as described above. Pupae were sexed according to size, introduced into cages and maintained during the adult stage at $27^{\circ} \mathrm{C}$, unless otherwise stated.

For assessing Wolbachia recovery during the adult stage, females from control and heat-treated groups were selected and divided into three different adult treatments: (i) C: control $\left(27^{\circ} \mathrm{C}\right.$ constant); (ii) adult temperature regime 1 (temperature peak at $32^{\circ} \mathrm{C}$ ); and (iii) adult temperature regime 2 (temperature peak at $37^{\circ} \mathrm{C}$ ). Temperature cycles for adult treatments are based on air temperature readings registered in Kuala Lumpur in February 2019. Readings for the adult temperature regime 1 were collected in the area of Pusat Komersial Shah Alam (303'57.2"N $\left.101^{\circ} 29^{\prime} 24.0^{\prime \prime E}\right)$ in a shaded location, while a semishaded area of the Institute of Medical Research ( $\left.3^{\circ} 10^{\prime} 10.3^{\prime \prime} \mathrm{N} 101^{\circ} 41^{\prime} 55.0^{\prime \prime} \mathrm{E}\right)$ was used for the adult regime 2.

Wolbachia density and fluorescent in situ hybridization. Genomic DNA from 5- to 7-day-old (unless otherwise stated) whole females and males of Wolbachia-carrying lines was extracted with STE buffer (10uM Tris HCL pH 8, $100 \mathrm{~mm} \mathrm{NaCl}, 1 \mathrm{~mm}$ EDTA) and used for Wolbachia density quantification by qPCR using the relative quantification of the Wolbachia surface protein (wsp) gene against the homothorax gene $(\mathrm{HTH})$ as reference gene. The following program was used to run the qPCRs: $95^{\circ} \mathrm{C}$ for $5 \mathrm{~min}$, $40 \times$ cycles of $95^{\circ} \mathrm{C}$ for $15 \mathrm{~s}$ and $60^{\circ} \mathrm{C}$ for $30 \mathrm{~s}$, followed by a melt-curve analysis. A Rotor Gene Q (Qiagen) was used with 2x QuantiNova SYBR.

Ovaries, salivary glands, and midguts (six pools of three organs per each replicate) were dissected from 5-day-old females using sterile forceps and needles in a drop of sterile PBS buffer, and immediately transferred into tubes containing STE buffer; genomic DNA from tissues was extracted and Wolbachia density was assessed by qPCR as previously described. Data shown in the plots are the representation of density quantitation of one of three independent biological replicates, consistently showing the same trend of results.

At the same time, ovaries were also dissected for FISH in sterile PBS buffer, and then immediately transferred to a tube containing Carnoy's fixative (chloroform:ethanol:acetic acid, 6:3:1) and fixed at $4^{\circ} \mathrm{C}$ overnight. Samples were then rinsed in PBS and incubated in a hybridization buffer containing: $50 \%$ formamide, $25 \% 20 \times \mathrm{SSC}, 0.2 \%$ (wt/vol) dextran sulphate, $2.5 \%$ herring sperm DNA, 1\% (wt/vol) tRNA, $0.015 \%$ (wt/vol) DTT, 1\% Denhardt's solution, and $100 \mathrm{ng} / \mathrm{ml}$ of each probe. The probes annealed on the wsp gene (5). Samples were left to hybridize overnight in a dark-humid box at $37^{\circ} \mathrm{C}$. Samples were washed twice in a solution containing: $5 \% 20 \times$ SSC, $0.015 \%$ (wt/vol) DTT, and twice in a solution of $2.5 \% \mathrm{SSC}, 0.015 \%$ (wt/vol) DTT in $\mathrm{dH} 2 \mathrm{O}$, and incubated at $55^{\circ} \mathrm{C}$ for $20 \mathrm{~min}$. Samples were then placed on a slide containing a drop of VECTASHIELD Antifade Mounting Medium with DAPI (Vector Laboratories, CA, USA) and were visualized immediately using a confocal microscope (ZEISS, Germany).

Wolbachia recovery during adult stage. After larval treatments, named control (Larval Control) and heat-treated (Larval Heat), eight females from different experimental groups denoted as larval-treatment/adult-treatment as follows: control/control; control/adult regime 1; control/adult regime 2; heat/ control; heat/adult regime 1 ; heat/adult regime 2 , were sampled after 0,7 , and 14 days. Midguts and salivary glands were also dissected a few hours after eclosion (day 0) and after 14 days. Wolbachia density was assessed in whole mosquitoes and tissues by qPCR as previously described.

Maternal transmission. Maternal transmission of each Wolbachia strain after heat stress was evaluated by backcrossing heat-treated females with heat-treated wild-type males, while control females mated with control wild-type males. After offering a blood-meal, 10 engorged females per group were selected and, after 3 days, individualized on damp circle of filter paper inside up-turned plastic cups. Filter papers were collected and individually desiccated. Once dried, eggs were hatched in containers and reared at stable control temperature; 6 to 10 fourth-instar larvae were randomly sampled from each individualized female (10 females) and assessed for Wolbachia infection by PCR, using strain specific primers described in Table S1. PCRs were set up using $1 \times$ Taqmaster mix (Vazyme) according to the manufacture's protocol; the amplification reaction consisted of a cycle at $94^{\circ} \mathrm{C}$ for $3 \mathrm{~min}$, followed by 30 cycles of denaturation at $94^{\circ} \mathrm{C}$ for $30 \mathrm{~s}$, annealing at $55^{\circ} \mathrm{C}$ for $30 \mathrm{~s}$, extension at $72^{\circ} \mathrm{C}$ for $30 \mathrm{~s}$, and a final step at $72^{\circ} \mathrm{C}$ for $10 \mathrm{~min}$. Additionally, a subset of samples (six individuals for six family) were validated by $\mathrm{qPCR}$, using wsp general primers.

Virus challenge. Five-day-old females per group were fed an infectious blood-meal consisting of human blood and DENV serotype-2 virus (New Guinea C Strain). The virus was serially passaged in Ae. albopictus C6/36 cells: the infected supernatant was harvested, concentrated using Amicon Ultra-15 filters (Millipore, IRL), and titered via fluorescent focus assay (FFA), as described below. Two independent challenges were carried out using different batches of propagated virus at the final concentration in the 
blood of $1.9 \times 10^{7} \mathrm{FFU} / \mathrm{ml}$ and $1.7 \times 10^{7} \mathrm{FFU} / \mathrm{ml}$, respectively. Fifty control and heat-treated females were infected for each challenge. Fully engorged females were transferred to a climatic chamber at $27^{\circ} \mathrm{C}, 70 \%$ relative humidity, and a 12 -h light/dark cycle, and maintained on $5 \%$ sucrose solution. After 12 days, mosquitoes were dissected and sampled: for the first challenge, salivary glands of control and heat-treated females were used to quantify virus titers, while only heat-treated individuals were involved for the second analysis. Samples were transferred in Dulbecco's Modified Eagle Medium (DMEM) supplemented with $2 \%$ fetal bovine serum (FBS). After homogenizing, 10-fold serial dilutions $\left(10^{-1}\right.$ to $\left.10^{-3}\right)$ of each solution was transferred onto a monolayer of Vero cells for viral quantification with FFA. Primary antibody for DENV was the MAB8705 anti-dengue virus complex antibody (Millipore); secondary antibody was the Goat anti-mouse Alexa Fluor 488, A-11001 (Thermo Scientific, Waltham, MA, USA). A Celigo Imaging Cytometer (Nexcelom Bioscience, Lawrence, MA, USA) was used for imaging plates. Fluorescent foci were counted by eye (from dilutions with less than $100 \mathrm{foci}$ ) and virus titers calculated and expressed as FFU/ml.

Statistical analysis. Graphics were generated using the ggplot2 package (50) of the $R$ software (version 3.6.1) (51) and Prism Software (version 9). Statistical tests were run using Prism version 9 and R. A Shapiro-Wilk test was used for assessing normality distribution of data, and parametric and nonparametric tests were selected accordingly for assessing Wolbachia density in mosquitoes. Wolbachia density data in the maternal transmission assay were analyzed with linear mixed models (Ime4 package) (52) after $\log _{10}$ transformation to meet the assumptions of normality. Post hoc pairwise multiple comparisons were performed with the function emmeans (R package emmeans) (53) and $P$ values adjusted using Bonferroni method.

Viral titers and infection prevalence were analyzed as the products of two independent processes of infection and amplification following infection using hurdle generalized linear models (GLM). The hurdle GLMs were composed of a zero-truncated negative binomial GLM conditioned on a binomial GLM and fitted using the $R$ package glmmTMB (54). Best-fitting models were selected by backward elimination of terms giving $P$ less than 0.05 starting from hurdle models with fixed effects of strain, heat treatment, and their interaction fitted in both binomial and negative binomial components. All $P$ values were calculated using likelihood ratio tests.

\section{SUPPLEMENTAL MATERIAL}

Supplemental material is available online only.

FIG S1, PDF file, $0.2 \mathrm{MB}$.

FIG S2, PDF file, $0.9 \mathrm{MB}$.

FIG S3, PDF file, $0.2 \mathrm{MB}$.

TABLE S1, PDF file, $0.1 \mathrm{MB}$.

\section{ACKNOWLEDGMENTS}

The study was supported by Wellcome Trust $(202888,108508)$ to SPS.

The funders had no role in study design, data collection, and analysis, decision to publish, or preparation of the manuscript.

We thank Ghazali M. R. Kamarul, Institute for Medical Research, Malaysia, for providing the temperature readings from Kuala Lumpur and Ary Hoffmann, University of Melbourne, for comments on the manuscript.

\section{REFERENCES}

1. Ross PA, Callahan AG, Yang Q, Jasper M, Arif MAK, Afizah AN, Nazni WA, Hoffmann AA. 2020. An elusive endosymbiont: Does Wolbachia occur naturally in Aedes aegypti? Ecol Evol 10:1581-1591. https://doi.org/10.1002/ ece3.6012.

2. Walker $\mathrm{T}$, Johnson $\mathrm{PH}$, Moreira LA, Iturbe-Ormaetxe I, Frentiu FD, McMeniman CJ, Leong YS, Dong Y, Axford J, Kriesner P, Lloyd AL, Ritchie SA, O'Neill SL, Hoffmann AA. 2011. The wMel Wolbachia strain blocks dengue and invades caged Aedes aegypti populations. Nature 476:450-453. https://doi.org/10.1038/nature10355.

3. Bian G, Xu Y, Lu P, Xie Y, Xi Z. 2010. The endosymbiotic bacterium Wolbachia induces resistance to dengue virus in Aedes aegypti. PLoS Pathog 6: e1000833. https://doi.org/10.1371/journal.ppat.1000833.

4. Hoffmann AA, Ross PA, Rašić G. 2015. Wolbachia strains for disease control: ecological and evolutionary considerations. Evol Appl 8:751-768. https://doi.org/10.1111/eva.12286.

5. Moreira LA, Iturbe-Ormaetxe I, Jeffery JA, Lu G, Pyke AT, Hedges LM, Rocha BC, Hall-Mendelin S, Day A, Riegler M, Hugo LE, Johnson KN, Kay $\mathrm{BH}, \mathrm{McGraw}$ EA, van den Hurk AF, Ryan PA, O'Neill SL. 2009. A Wolbachia symbiont in Aedes aegypti limits infection with dengue, chikungunya, and plasmodium. Cell 139:1268-1278. https://doi.org/10.1016/j.cell.2009.11 042 .

6. Tan $\mathrm{CH}$, Wong PJ, Li MI, Yang H, Ng LC, O'Neill SL. 2017. wMel limits zika and chikungunya virus infection in a Singapore Wolbachia-introgressed Ae. aegypti strain, wMel-Sg. PLoS Negl Trop Dis 11:e0005496. https://doi .org/10.1371/journal.pntd.0005496.

7. Ant TH, Herd CS, Geoghegan V, Hoffmann AA, Sinkins SP. 2018. The Wolbachia strain wAu provides highly efficient virus transmission blocking in Aedes aegypti. PLoS Pathog 14:e1006815. https://doi.org/10.1371/journal .ppat. 1006815 .

8. Nazni WA, Hoffmann AA, NoorAfizah A, Cheong YL, Mancini MV, Golding $\mathrm{N}$, Kamarul GMR, Arif MAK, Thohir H, NurSyamimi H, ZatilAqmar MZ, NurRuqqayah M, NorSyazwani A, Faiz A, Irfan FMN, Rubaaini S, Nuradila N, Nizam NMN, Irwan SM, Endersby-Harshman NM, White VL, Ant TH, Herd CS, Hasnor AH, AbuBakar R, Hapsah DM, Khadijah K, Kamilan D, Lee SC, Paid YM, Fadzilah K, Topek O, Gill BS, Lee HL, Sinkins SP. 2019. Establishment of Wolbachia strain wAlbB in Malaysian populations of Aedes aegypti for dengue control. Curr Biol 29:4241-4248. https://doi.org/10.1016/j.cub .2019.11.007. 
9. Ryan PA, Turley AP, Wilson G, Hurst TP, Retzki K, Brown-Kenyon J, Hodgson L, Kenny N, Cook H, Montgomery BL, Paton CJ, Ritchie SA, Hoffmann AA, Jewell NP, Tanamas SK, Anders KL, Simmons CP, O'Neill SL. 2019. Establishment of WMel Wolbachia in Aedes aegypti mosquitoes and reduction of local dengue transmission in Cairns and surrounding locations in northern Queensland, Australia. Gates Open Res 3:1547. https:// doi.org/10.12688/gatesopenres.13061.1.

10. Tantowijoyo W, Andari B, Arguni E, Budiwati N, Nurhayati I, Fitriana I, Ernesia I, Daniwijaya EW, Supriyati E, Yusdiana DH, Victorius M, Wardana DS, Ardiansyah H, Ahmad RA, Ryan PA, Simmons CP, Hoffmann AA, Rancès E, Turley AP, Johnson P, Utarini A, O'Neill SL. 2020. Stable establishment of wMel Wolbachia in Aedes aegypti populations in Yogyakarta, Indonesia. PLoS Negl Trop Dis 14:e0008157. https://doi.org/10.1371/ journal.pntd.0008157.

11. Joubert DA, Walker T, Carrington LB, De Bruyne JT, Kien DHT, Hoang NLT, Chau NVV, Iturbe-Ormaetxe I, Simmons CP, O'Neill SL. 2016. Establishment of a Wolbachia superinfection in Aedes aegypti mosquitoes as a potential approach for future resistance management. PLoS Pathog 12: e1005434. https://doi.org/10.1371/journal.ppat.1005434.

12. Fraser JE, De Bruyne JT, Iturbe-Ormaetxe I, Stepnell J, Burns RL, Flores HA, O'Neill SL. 2017. Novel Wolbachia-transinfected Aedes aegypti mosquitoes possess diverse fitness and vector competence phenotypes. PLoS Pathog 13:e1006751. https://doi.org/10.1371/journal.ppat.1006751.

13. Teixeira L, Ferreira A, Ashburner M. 2008. The bacterial symbiont Wolbachia induces resistance to RNA viral infections in drosophila melanogaster. PLoS Biol 6:e2. https://doi.org/10.1371/journal.pbio.1000002.

14. Hedges LM, Brownlie JC, O'Neill SL, Johnson KN. 2008. Wolbachia and virus protection in insects. Science 322:702. https://doi.org/10.1126/ science.1162418.

15. Dutra HL, Rocha MN, Dias FB, Mansur SB, Caragata EP, Moreira LA. 2016. Wolbachia blocks currently circulating zika virus isolates in Brazilian Aedes aegypti mosquitoes. Cell Host Microbe 19:771-774. https://doi.org/10 .1016/j.chom.2016.04.021.

16. O’Neill SL, Ryan PA, Turley AP, Wilson G, Retzki K, Iturbe-Ormaetxe I, Dong Y, Kenny N, Paton CJ, Ritchie SA, Brown-Kenyon J, Stanford D, Wittmeier N, Jewell NP, Tanamas SK, Anders KL, Simmons CP. 2018. Scaled deployment of Wolbachia to protect the community from dengue and other Aedes transmitted arboviruses. Gates Open Res 2:36. https://doi.org/10 .12688/gatesopenres.12844.1.

17. Indriani C, Tantowijoyo W, Rancès E, Andari B, Prabowo E, Yusdi D, Ansari MR, Wardana DS, Supriyati E, Nurhayati I, Ernesia I, Setyawan S, Fitriana I, Arguni E, Amelia Y, Ahmad RA, Jewell NP, Dufault SM, Ryan PA, Green BR, McAdam TF, O'Neill SL, Tanamas SK, Simmons CP, Anders KL, Utarini A. 2020. Reduced dengue incidence following deployments of Wolbachiainfected Aedes aegypti in Yogyakarta, Indonesia: a quasi-experimental trial using controlled interrupted time series analysis. Gates Open Res 4: 50. https://doi.org/10.12688/gatesopenres.13122.1.

18. Utarini A, Indriani C, Ahmad RA, Tantowijoyo W, Arguni E, Ansari MR, Supriyati E, Wardana DS, Meitika Y, Ernesia I, Nurhayati I, Prabowo E, Andari B, Green BR, Hodgson L, Cutcher Z, Rancès E, Ryan PA, O'Neill SL, Dufault SM, Tanamas SK, Jewell NP, Anders KL, Simmons CP, AWED Study Group. 2021. Efficacy of Wolbachia-infected mosquito deployments for the control of dengue. N Engl J Med 384:2177-2186. https://doi.org/10 .1056/NEJMoa2030243.

19. Ekwudu O, Devine GJ, Aaskov JG, Frentiu FD. 2020. Wolbachia strain wAlbB blocks replication of flaviviruses and alphaviruses in mosquito cell culture. Parasit Vectors 13:54. https://doi.org/10.1186/s13071-020-3936-3.

20. Ahmad NA, Mancini MV, Ant TH, Martinez J, Kamarul GMR, Nazni WA, Hoffmann AA, Sinkins SP. 2021. Wolbachia strain wAlbB maintains high density and dengue inhibition following introduction into a field population of Aedes aegypti. Philos Trans R Soc Lond B Biol Sci 376:20190809. https://doi.org/10.1098/rstb.2019.0809.

21. Martinez J, Longdon B, Bauer S, Chan YS, Miller WJ, Bourtzis K, Teixeira L, Jiggins FM. 2014. Symbionts commonly provide broad spectrum resistance to viruses in insects: a comparative analysis of Wolbachia strains. PLoS Pathog 10:e1004369. https://doi.org/10.1371/journal.ppat.1004369.

22. Lu P, Bian G, Pan X, Xi Z. 2012. Wolbachia induces density-dependent inhibition to dengue virus in mosquito cells. PLoS Negl Trop Dis 6:e1754. https://doi.org/10.1371/journal.pntd.0001754.

23. McMeniman CJ, Lane RV, Cass BN, Fong AW, Sidhu M, Wang YF, O'Neill SL. 2009. Stable introduction of a life-shortening Wolbachia infection into the mosquito Aedes aegypti. Science 323:141-144. https://doi.org/10 $.1126 /$ science. 1165326 .
24. Min KT, Benzer S. 1997. Wolbachia, normally a symbiont of Drosophila, can be virulent, causing degeneration and early death. Proc Natl Acad Sci USA 94:10792-10796. https://doi.org/10.1073/pnas.94.20.10792.

25. Chrostek E, Marialva MS, Yamada R, O’Neill SL, Teixeira L. 2014. High antiviral protection without immune upregulation after interspecies Wolbachia transfer. PLoS One 9:e99025. https://doi.org/10.1371/journal.pone .0099025 .

26. Fraser JE, O'Donnell TB, Duyvestyn JM, O'Neill SL, Simmons CP, Flores HA. 2020. Novel phenotype of Wolbachia strain wPip in Aedes aegypti challenges assumptions on mechanisms of Wolbachia-mediated dengue virus inhibition. PLoS Pathog 16:e1008410. https://doi.org/10.1371/journal .ppat. 1008410.

27. Axford JK, Ross PA, Yeap HL, Callahan AG, Hoffmann AA. 2016. Fitness of wAlbB Wolbachia infection in Aedes aegypti: parameter estimates in an outcrossed background and potential for population invasion. Am J Trop Med Hyg 94:507-516. https://doi.org/10.4269/ajtmh.15-0608.

28. Hancock PA, White VL, Ritchie SA, Hoffmann AA, Godfray HC. 2016. Predicting Wolbachia invasion dynamics in Aedes aegypti populations using models of density-dependent demographic traits. BMC Biol 14:96. https://doi.org/10.1186/s12915-016-0319-5.

29. Barton NH, Turelli M. 2011. Spatial waves of advance with bistable dynamics: cytoplasmic and genetic analogues of allee effects. Am Nat 178: E48-75. https://doi.org/10.1086/661246.

30. Nguyen TH, Nguyen HL, Nguyen TY, Vu SN, Tran ND, Le TN, Vien QM, Bui TC, Le HT, Kutcher S, Hurst TP, Duong TT, Jeffery JA, Darbro JM, Kay BH, IturbeOrmaetxe I, Popovici J, Montgomery BL, Turley AP, Zigterman F, Cook $\mathrm{H}_{\text {, }}$ Cook PE, Johnson PH, Ryan PA, Paton CJ, Ritchie SA, Simmons CP, O'Neill SL, Hoffmann AA. 2015. Field evaluation of the establishment potential of $w$ MelPop Wolbachia in Australia and Vietnam for dengue control. Parasit Vectors 8:563. https://doi.org/10.1186/s13071-015-1174-x.

31. Wernegreen JJ. 2012. Mutualism meltdown in insects: bacteria constrain thermal adaptation. Curr Opin Microbiol 15:255-262. https://doi.org/10 .1016/j.mib.2012.02.001.

32. Corbin C, Heyworth ER, Ferrari J, Hurst GD. 2017. Heritable symbionts in a world of varying temperature. Heredity (Edinb) 118:10-20. https://doi .org/10.1038/hdy.2016.71.

33. Sazama EJ, Ouellette SP, Wesner JS. 2019. Bacterial endosymbionts are common among, but not necessarily within, insect species. Environ Entomol 48:127-133. https://doi.org/10.1093/ee/nvy188.

34. Sumi T, Miura K, Miyatake T. 2017. Wolbachia density changes seasonally amongst populations of the pale grass blue butterfly, Zizeeria maha (Lepidoptera: Lycaenidae). PLoS One 12:e0175373. https://doi.org/10.1371/ journal.pone.0175373.

35. Ross PA, Axford JK, Yang Q, Staunton KM, Ritchie SA, Richardson KM, Hoffmann AA. 2020. Heatwaves cause fluctuations in wMel Wolbachia densities and frequencies in Aedes aegypti. PLoS Negl Trop Dis 14: e0007958. https://doi.org/10.1371/journal.pntd.0007958.

36. Ross PA, Wiwatanaratanabutr I, Axford JK, White VL, Endersby-Harshman NM, Hoffmann AA. 2017. Wolbachia infections in Aedes aegypti differ markedly in their response to cyclical heat stress. PLoS Pathog 13: e1006006. https://doi.org/10.1371/journal.ppat.1006006.

37. Ulrich JN, Beier JC, Devine GJ, Hugo LE. 2016. Heat sensitivity of wMel Wolbachia during Aedes aegypti development. PLoS Negl Trop Dis 10: e0004873. https://doi.org/10.1371/journal.pntd.0004873.

38. Hemme RR, Tank JL, Chadee DD, Severson DW. 2009. Environmental conditions in water storage drums and influences on Aedes aegypti in Trinidad, West Indies. Acta Trop 112:59-66. https://doi.org/10.1016/j.actatropica.2009 .06.008.

39. Ross PA, Hoffmann AA. 2018. Continued Susceptibility of the wMel Wolbachia infection in Aedes aegypti to heat stress following field deployment and selection. Insects 9:78. https://doi.org/10.3390/insects9030078.

40. Paaijmans KP, Jacobs AFG, Takken W, Heusinkveld BG, Githeko AK, Dicke M, Holtslag AAM. 2008. Observations and model estimates of diurnal water temperature dynamics in mosquito breeding sites in western Kenya. Hydrol Process 22:4789-4801. https://doi.org/10.1002/hyp.7099.

41. Nainu F, Trenerry A, Johnson KN. 2019. Wolbachia-mediated antiviral protection is cell-autonomous. J Gen Virol 100:1587-1592. https://doi.org/10 .1099/jgv.0.001342.

42. Cattarino L, Rodriguez-Barraquer I, Imai N, Cummings DAT, Ferguson NM. 2020. Mapping global variation in dengue transmission intensity. Sci Transl Med 12: eaax4144. https://doi.org/10.1126/scitranslmed.aax4144.

43. Geoghegan V, Stainton K, Rainey SM, Ant TH, Dowle AA, Larson T, Hester S, Charles PD, Thomas B, Sinkins SP. 2017. Perturbed cholesterol and vesicular trafficking associated with dengue blocking in Wolbachia-infected 
Aedes aegypti cells. Nat Commun 8:526. https://doi.org/10.1038/s41467 $-017-00610-8$

44. Schmidt TL, Barton NH, Rašić G, Turley AP, Montgomery BL, IturbeOrmaetxe I, Cook PE, Ryan PA, Ritchie SA, Hoffmann AA, O'Neill SL, Turelli M. 2017. Local introduction and heterogeneous spatial spread of denguesuppressing Wolbachia through an urban population of Aedes aegypti. PLoS Biol 15:e2001894. https://doi.org/10.1371/journal.pbio.2001894.

45. Garcia GA, Sylvestre G, Aguiar R, da Costa GB, Martins AJ, Lima JBP, Petersen MT, Lourenco-de-Oliveira R, Shadbolt MF, Rasic G, Hoffmann AA, Villela DAM, Dias FBS, Dong Y, O'Neill SL, Moreira LA, Maciel-de-Freitas R. 2019. Matching the genetics of released and local Aedes aegypti populations is critical to assure Wolbachia invasion. PLoS Negl Trop Dis 13: e0007023. https://doi.org/10.1371/journal.pntd.0007023.

46. Carrington LB, Tran BCN, Le NTH, Luong TTH, Nguyen T, Nguyen PT, Nguyen CVV, Nguyen HTC, Vu TT, Vo LT, Le DT, Vu NT, Nguyen GT, Luu $H Q$, Dang AD, Hurst TP, O'Neill SL, Tran VT, Kien DTH, Nguyen NM, Wolbers M, Wills B, Simmons CP. 2018. Field- and clinically derived estimates of Wolbachia-mediated blocking of dengue virus transmission potential in Aedes aegypti mosquitoes. Proc Natl Acad Sci USA 115: 361-366. https://doi.org/10.1073/pnas.1715788115.
47. Ye YH, Carrasco AM, Dong Y, Sgrò CM, McGraw EA. 2016. The effect of temperature on Wolbachia-mediated dengue virus blocking in Aedes aegypti. Am J Trop Med Hyg 94:812-819. https://doi.org/10.4269/ajtmh .15-0801.

48. Chrostek E, Martins N, Marialva MS, Teixeira L. 2021. Conferred antiviral protection is determined by developmental temperature. mBio 7:e0292320. https://doi.org/10.1128/mBio.02923-20.

49. Reinhold JM, Lazzari CR, Lahondère C. 2018. Effects of the environmental temperature on insects. Insects 9:158. https://doi.org/10.3390/insects9040158.

50. Hadley W. 2016. ggplot2: Elegant graphics for data analysis. Springer-Verlag New York.

51. R Core Team. 2021. R: A language and environment for statistical computing. R Foundation for Statistical Computing, Vienna, Austria.

52. Bates D, Mächler M, Bolker B, Walker S. 2015. Fitting linear mixed-effects models using \{lme4\}. J Stat Soft 67:48. https://doi.org/10.18637/jss.v067.i01.

53. Lenth R. 2019. Emmeans: Estimated marginal means, aka least-squares means. https://CRAN.Rproject.org/package=emmeans.

54. Brooks M, Kristensen E, Benthem K, van Magnusson KJ, Berg A, Nielsen CW, Skaug A, Mächler HJ, Bolker M, Benjamin M. 2017. glmmTMB balances speed and flexibility among packages for zero-inflated generalized linear mixed modeling. The R J 9:378-400. https://doi.org/10.32614/RJ-2017-066. 